\title{
A NOVEL ROLE FOR THE GAS3/PMP22 FAMILY MEMBER EMP2 IN THE REGULATION OF INFLAMMATION
}

M Daigneault, A Angyal, E Hadadi, J Baskerville, H Wilson University of Sheffield

doi:10.1136/heartjnl-2013-304019.208

Introduction Cardiovascular disease such as atherosclerosis is currently the leading cause of death by noncommunicable diseases worldwide. Development of atherosclerosis which is considered a chronic inflammatory disease has been associated with a number of pro-inflammatory cytokines including interleukin-1 (IL-1). IL-1 has been associated with atherosclerotic plaque formation as well as plaque rupture. Previous reports using $\mathrm{ApoE}^{-/-} / \mathrm{IL} 1-\beta^{-/-}$mice have described a significant decrease in atherosclerotic area further highlighting the importance of this apical cytokine. IL-1 $\beta$ is released by monocytes and macrophages following P2X7 receptor activation by ATP, however the exact mechanism by which release occurs is poorly understood. We have previously identified the GAS3/PMP22 family member, epithelial membrane protein-2 
(EMP2), as a P2X7 C-terminus interacting protein. Blocking EMP2 has been shown to reduce early Chlamydia trachomatis infectivity and modify cytokine secretion; however the function of EMP2 in this role is not described. The purpose of this study was to establish the role of EMP2 in P2X7 receptor dependent IL-1 $\beta$ release.

Methods THP1 monocytic cells were transfected with nontargeting control siRNA, siRNA specific for EMP2 or the fluorescent indicator SiGLO, using the reagent Dharmafect Duo with an optimised protocol for THP-1 cells. Transfection efficiency was determined by flow cytometry and the efficiency of the knockdown was assessed by real-time PCR. THP1 cells were then treated with PMA (500 nM) for 3 hours to promote differentiation to a more macrophage like phenotype. Differentiated THP1 cells were stimulated with $1 \mu \mathrm{g} / \mathrm{ml}$ of LPS with and without a P2X7 receptor antagonist (A438079 hydrochloride), followed by BzATP (300 $\mu \mathrm{M}$, P2X7 agonist) for 20 minutes. Cell supernatants were collected and IL-1 $\beta$ release was measured by ELISA; cytotoxicity was determined by lactate dehydrogenase (LDH) release.

Results BzATP treatment of THP1 cells significantly enhanced IL-1 $\beta$ release compared with LPS stimulation alone. P2X7 receptor dependent IL-1 $\beta$ release was almost completely inhibited by pretreatment with the receptor antagonist. SiRNA knockdown of EMP2 was approximately $40 \%$, as confirmed by real-time PCR, and significantly enhanced P2X7 receptor dependent IL-1 $\beta$ release compared with controls. There was no significant difference in LDH release following stimulation with LPS or BzATP, suggesting that the increase in IL-1 $\beta$ release was not due to cytotoxicity.

Conclusions EMP2 contributes to the regulation of $\mathrm{P} 2 \mathrm{X} 7$ receptor dependent IL-1 $\beta$ release by differentiated THP1 cells. 